\title{
Efeito de uma multimistura alimentar no estado nutricional relativo ao ferro em pré-escolares
}

\author{
Effect of a multi-mixture in the nutritional status \\ of preschool children regarding iron
}

Luciana Ferreira da Rocha SANT'ANA ${ }^{1}$

Ana Cristina Rodrigues Ferreira da CRUZ²

Sylvia do Carmo Castro FRANCESCHINI ${ }^{1}$

Neuza Maria Brunoro COSTA'

RE S U M O

\section{Objetivo}

Avaliar o efeito do consumo de uma multimistura alimentar no estado nutricional relativo ao ferro em pré-escolares da rede municipal de ensino em Viçosa, MG.

\section{Métodos}

Participaram 36 crianças, distribuídas em três creches da cidade. Na creche A foram fornecidos produtos de panificação sem acréscimo de ferro. Na creche $B$, produtos acrescidos de sulfato ferroso em quantidade equivalente ao teor de ferro fornecido pelas preparações com multimistura oferecidas na terceira creche. Os produtos foram distribuídos pela manhã, durante 70 dias. Foram realizadas avaliações antropométricas, dietéticas e bioquímicas no início e no final do estudo, pelo teste " $t$ " pareado a $5 \%$ de probabilidade, exceto para o hematócrito. Neste caso, os valores iniciais foram comparados aos finais pelo teste de Wilcoxon, a 5\% de probabilidade. As análises foram feitas utilizando-se o programa Sistema de Análises Estatísticas e Genética, versão 5.0, da Universidade Federal de Viçosa.

\section{Resultados}

Não foi demonstrado efeito das diferentes fontes de ferro, dado que as creches suplementadas apresentaram redução dos níveis hematológicos avaliados, o que não ocorreu com a creche A (sem adição de ferro).

\section{Conclusão}

O ferro fornecido como suplementação não alterou a ingestão dietética de ferro pelas crianças e nem melhorou os índices hematológicos avaliados, limitando as conclusões quanto à utilização da multimistura em estudo.

Termos de indexação: biodisponibilidade; estado nutricional; ferro; multimistura; pré-escolar.

\footnotetext{
1 Departamento de Nutrição e Saúde, Universidade Federal de Viçosa. Av. P.H. Rolfs, s/n, Campus Universitário, 36571-000, Viçosa, MG, Brasil. Correspondência para/Correspondence to: N.M.B. COSTA.E-mail: <nmbc@ufv.br>.

2 Mestre em Ciência da Nutrição, Universidade Federal de Viçosa. Viçosa, MG, Brasil.
} 


\section{A B S T R A C T}

\section{Objective}

The purpose of this study was to evaluate the effect of a multi-mixture intake on the iron status of pre-school children from the municipal school network of Viçosa, Minas Gerais, Brazil.

\section{Methods}

Thirty-six children from three day nurseries of the city participated in the study. Day nursery A received bakery products not supplemented with iron. Day nursery B received bakery products supplemented with iron sulfate in the same amount as that present in multi-mixtures distributed in the third day nursery. The products were distributed in the morning during 70 days. Anthropometric, dietary and biochemical assessments were done at the beginning and at the end of the study using the paired t-test at significance level of 0.05, except for hematocrit. In this case, the initial values were compared with the final values using the Wilcoxon test at significance level of 0.05. The software Statistical Analyses and Genetics System, version 5.0, from the Federal University of Viçosa was used for the analyses.

\section{Results}

The different sources of iron had no effect since the supplemented groups showed a reduction in the assessed blood levels and this was not observed in the placebo group.

\section{Conclusion}

The iron given as a supplement did not alter the iron intake by the children and did not improve the assessed blood indexes either, limiting the conclusions of using the multi-mixture studied in this work.

Indexing terms: biological availability; nutritional status; iron; nutritional suplement; child, preschool.

\section{N T R O D U ÇÃ O}

A deficiência de ferro é considerada a carência nutricional mais prevalente em todo o mundo, afetando, principalmente, lactentes, pré-escolares, adolescentes e gestantes. A anemia, por diminuição anormal na concentração de hemoglobina no sangue, é considerada a principal conseqüência da deficiência de ferro. Em sua fase mais avançada, está associada a sintomas clínicos, como fraqueza, diminuição da capacidade respiratória e tontura. Mesmo na ausência de anemia, a deficiência de ferro pode acarretar distúrbios neurológicos ${ }^{1,2}$, às vezes com conseqüências irreversíveis ${ }^{3}$.

A anemia por deficiência de ferro é um processo evolutivo que se inicia com a depleção dos estoques de ferro, passa pela queda no ferro em transporte e termina na redução do ferro ligado à hemoglobina, resultando na anemia clínica ${ }^{4,5}$.

No Brasil, o sulfato ferroso, ferro inorgânico não hematínico, é a fonte de ferro mais utilizada para a suplementação. Além de poder causar efeitos colaterais, sua absorção está comprome- tida na presença de fatores dietéticos, assim como ocorre com o ferro de origem vegetal ${ }^{3,6}$. Várias são as tentativas de fornecer, por meio da dieta, a quota de ferro necessária. Alimentos alternativos, como farelos de cereais, folhas verde-escuras, sementes e cascas de frutas, têm sido introduzidos na dieta habitual como uma forma de enriquecê-la. Esses alimentos não-convencionais, em sua maioria de origem vegetal, formam as multimisturas, que são oferecidas em pequenas quantidades ao dia, com o intuito de fornecer os minerais e vitaminas que são perdidos durante $o$ processamento ou beneficiamento de alimentos ${ }^{7}$. No Brasil, a alimentação alternativa visa, entre outras coisas, recuperar o estado nutricional de ferro em crianças, fornecendo-lhes multimisturas.

Sant'Ana et al. ${ }^{8}$, estudando o valor nutritivo de alguns desses alimentos, verificaram que são fontes potenciais de minerais, como o ferro, cálcio e zinco, entretanto pouco, ou quase nada, se sabe sobre sua biodisponibilidade, que pode estar comprometida pela presença de fitatos, oxalatos e inibidor de tripsina. 
Considerando que as multimisturas são alimentos não-convencionais de origem vegetal, que trazem quantidades relevantes de minerais e também de antinutrientes, este trabalho teve como objetivo avaliar o efeito da adição de uma multimistura alimentar no estado nutricional relativo ao ferro em pré-escolares da rede municipal de ensino do município de Viçosa, MG.

\section{MÉTODOS}

A multimistura utilizada no estudo era composta por $33 \%$ de farinha de trigo, $33 \%$ de fubá de milho, $32 \%$ de farelo de trigo, $1 \%$ de pó de folha de mandioca e $1 \%$ de pó de casca de ovo. A farinha de trigo e o fubá de milho, adquiridos no comércio local, foram acrescentados crus à mistura. O farelo de trigo, também adquirido no comércio local, foi torrado previamente à sua incorporação à multimistura. As folhas de mandioca, adquiridas já desidratadas, foram processadas, em multiprocessador doméstico, para obtenção do pó. As cascas de ovo de galinha foram obtidas em uma panificadora local e processadas de acordo com o manual da alimentação alternativa ${ }^{7}$. Depois de preparar os ingredientes, esses foram misturados nas proporções supracitadas para, então, constituírem a multimistura avaliada nesta pesquisa.

O protocolo foi aprovado pelo Comitê de Ética na Pesquisa com Seres Humanos da Universidade Federal de Viçosa e, posteriormente, pela Secretaria de Educação do município de Viçosa, MG. Em reunião em três creches, escolhidas aleatoriamente, foram esclarecidos os objetivos do trabalho e sua forma de execução para professores, monitores, pais e responsáveis, sendo, na ocasião, assinado um termo de consentimento e autorização para que as crianças que nelas estudavam participassem do estudo.

$\mathrm{Na}$ etapa inicial foram recrutadas 53 crianças, de 25 a 68 meses de idade, distribuídas em três creches da rede municipal de ensino de Viçosa, MG. Essas creches funcionavam em regime de oito horas, fornecendo, nesse período, três refeições: lanche da manhã, almoço e lanche da tarde, de segunda a sexta-feira.

Entre outros critérios, o termo de consentimento previu que as crianças deveriam ser voluntárias, ter índices normais de hemoglobina $(\geq 11,0 \mathrm{~g} / \mathrm{dl})$ e consumir satisfatoriamente os produtos oferecidos, tanto em quantidade como em freqüência de consumo ${ }^{9}$. Para tanto, as crianças deveriam consumir toda a porção do produto oferecido no dia e ser freqüentes na creche durante o estudo. Em virtude desses critérios, houve variações no número de crianças participantes nas diversas análises realizadas.

As crianças foram submetidas a avaliações dietética, antropométrica e bioquímica, no início e final do estudo.

Para avaliação do consumo quantitativo de alimentos que a criança ingeriu no período em que estava na creche, foi utilizado o método de pesagem direta, durante três dias da semana, consecutivos. Pesou-se cada porção dos alimentos que compunham as três refeições servidas nas creches em balança digital, portátil, com capacidade para $2 \mathrm{~kg}$ e divisão de $1 \mathrm{~g}$. Também foi feita a observação direta do consumo das crianças, no momento da distribuição das refeições.

Por meio do método recordatório de 24 horas ${ }^{10}$, foram obtidas as informações dos responsáveis pelas refeições realizadas pelas crianças fora das creches nesses mesmos dias, com base na "Tabela para avaliação de consumo alimentar em medidas caseiras"11. As análises e os cálculos das dietas foram feitos utilizando-se o software DIETPRO, versão 2.012. Após a análise do consumo de nutrientes diário foi feita uma média deste consumo para avaliação dietética de cada criança estudada.

As crianças foram avaliadas antropometricamente quanto ao peso e à estatura. Foram feitas análises dos índices peso para idade (P/I), peso para estatura (P/E) e estatura para idade (E/I), utilizando-se como critério diagnóstico o z-score. Considerou-se, nessas análises, a refe- 
rência antropométrica do National Center for Health Statistics $(\mathrm{NCHS})^{13}$, por meio do software Epi Info, versão $6.01^{14}$.

Como ponto de corte para desnutrição utilizou-se o critério de $<-2 z$ para o índice peso/idade e, ou, peso/estatura ${ }^{13}$. Foram considerados como risco para desnutrição o peso/idade e, ou, o peso/estatura no intervalo entre -1 e $-2 z$. O ponto de corte de $<-2 z$ foi também utilizado para o diagnóstico de baixa estatura, considerando-se o índice estatura/idade. As crianças que se encontravam entre -1 e $-2 z$ foram classificadas como tendo déficit estatural. Ainda, foram consideradas com sobrepeso aquelas crianças com os índices peso/idade e, ou, peso/estatura acima de $+2 z$. As crianças que apresentaram tais índices, entre +1 e $+2 z$, foram classificadas como em risco de sobrepeso.

Para a medida de peso foi utilizada uma balança portátil, digital e eletrônica, com capacidade para $150 \mathrm{~kg}$ e divisão de $50 \mathrm{~g}$. As medidas de altura foram feitas com o uso de fita métrica metálica (Microtoise - Stanley - Mabo Ltda), com extensão de $2 \mathrm{~m}$ e divisão de $1 \mathrm{~mm}$. As técnicas utilizadas para obtenção de peso e altura foram as propostas por Jellife ${ }^{15}$.

Os exames bioquímicos também foram realizados entre agosto e novembro de 2001 . A coleta de sangue das crianças foi feita na creche em que estudavam, após jejum de 10 horas, por um bioquímico, auxiliado por um técnico de enfermagem. Foram colhidas, em seringas descartáveis, por punção venosa, amostras de $6 \mathrm{ml}$ de sangue. Cerca de $3 \mathrm{ml}$ da amostra colhida eram transferidos para um tubo com anticoagulante EDTA, para realização do eritrograma (contagem de hemácias, hematócrito e concentração de hemoglobina); e o restante do sangue foi transferido para um tubo sem anticoagulante, para obtenção do soro, no qual foram determinados os níveis de ferritina e ferro séricos.

O eritrograma foi realizado em aparelho Cobas Argos, Roche. O ferro sérico foi determinado pelo método químico de Goodwin, modificado (Ferrozine), utilizando kit da marca in vitro diagnóstica; e a ferritina sérica foi determinada por quimioluminescência, em aparelho Immulite 1000, DPC Medilab.

As análises hematológicas foram realizadas em laboratório de análises clínicas de referência no município de Viçosa, MG.

O estudo foi realizado em três creches municipais, cada uma constituindo um grupo experimental. Na creche $A$, as crianças receberam os produtos de panificação sem acréscimo de ferro $(n=9)$. Na creche $B(n=12)$, elas receberam os produtos acrescidos de sulfato ferroso em quantidade equivalente ao teor de ferro fornecido pelas preparações com multimistura. E, na creche C (multimistura, $n=15)$, as crianças receberam produtos, cuja multimistura era a fonte de ferro. A multimistura avaliada apresentava um conteúdo de cerca de 5,3mg Fe/100g. É importante ressaltar que as creches $B$ e $C$ receberam quantidade diária de ferro dietético, provenientes dos produtos testados, em acréscimo ao total consumido normalmente. Esse teor de ferro fornecido foi baseado na recomendação ${ }^{7}$ do consumo médio diário, por pessoa, de duas colheres de sopa de multimistura, o que fornece cerca de $1 \mathrm{mg}$ de ferro/dia, correspondendo a $10 \%$ das necessidades de ferro para crianças na mesma faixa etária que as deste estudo ${ }^{16}$

A intervenção se deu pelo fornecimento de produtos de panificação (bolos e biscoitos) servidos às crianças durante 70 dias, de segunda a sexta-feira, no lanche da manhã. Esse lanche foi modificado, sendo os biscoitos usualmente servidos substituídos pelos produtos testados. No Quadro 1, mostra-se o teor de ferro programado para os produtos confeccionados que serviram de veículo das fontes desse mineral.

As receitas dos produtos anteriormente indicados eram elaboradas todo dia, segundo Ferreira et al. ${ }^{17}$, no Laboratório de Estudo Experimental dos Alimentos, do Departamento de Nutrição e Saúde da Universidade Federal de Viçosa. Os produtos eram transportados individualmente em embalagens de isopor, contendo a identificação da criança. A distribuição era diária 
(de $2^{a}$ a $6^{\text {a }}$ feira), e cada criança recebia a sua porção.

Várias crianças $(32,1 \%)$ foram excluídas da amostra por não terem realizado o segundo exame hematológico. Assim, 36 crianças entre as 53 na amostra original, com níveis normais de hemoglobina, participaram da avaliação final do estudo.

O banco de dados foi montado utilizando-se o Programa Epi Info, versão 6.01 b ${ }^{14}$.

Foi feita uma distribuição aleatória dos tratamentos entre as creches. Para analisar o período de intervenção, os dados coletados no início do experimento (antropométricos, dietéticos e bioquímicos), foram comparados com os obtidos no final do estudo, pelo teste " $t$ " de Student, pareado a $5 \%$ de probabilidade ${ }^{18}$, exceto o hematócrito. Nesse caso, os valores iniciais foram comparados com os finais pelo teste de Wilcoxon a $5 \%$ de probabilidade ${ }^{19}$.

Foram analisados os índices antropométricos peso/estatura, peso/idade e estatura/ idade; o consumo de energia, carboidratos, lipídios, proteínas, fibra, ferro, cálcio, zinco, cobre,

Quadro 1. Produtos de panificação (bolos e biscoitos) servidos nas creches em estudo, com os respectivos teores de ferro (mg/100g pronto), em Viçosa, 2001.

\begin{tabular}{lcc}
\hline \multirow{2}{*}{ Produtos de panificação } & \multicolumn{2}{c}{ Teor de Ferro (mg/100g pronto) } \\
\cline { 2 - 3 } & $\begin{array}{c}\text { Sem acréscimo } \\
\text { de ferro }\end{array}$ & $\begin{array}{c}\text { Com acréscimo de } \\
\text { ferro (FeSO } \\
\text { multimistura) }\end{array}$ \\
\hline Pirulito do chaves & 1,34 & 2,91 \\
Brevidade & 0,41 & 2,60 \\
Pão de mel & 0,75 & 1,72 \\
Bolinho surpresa & 0,69 & 2,03 \\
Limãozinho & 0,69 & 1,54 \\
Cookie tricolor & 0,92 & 2,10 \\
Biscoito da minie & 1,17 & 2,77 \\
Goiabinha & 0,72 & 2,26 \\
Biscoito surpresa & 0,00 & 2,31 \\
Biscoito de coco & 0,84 & 2,76 \\
Bolo de mandioca & 0,50 & 1,74 \\
Bolo de banana & 0,95 & 2,20 \\
Moranguinho & 0,69 & 1,55 \\
Biscoito de chocolate & 1,32 & 2,28 \\
Biscoito de leite em pó & 0,29 & 2,02 \\
\hline
\end{tabular}

vitaminas A e C; e os parâmetros hematológicos e bioquímicos: hemoglobina, hematócrito, hemácias, ferritina e ferro sérico. As análises estatísticas foram feitas utilizando-se o programa Sistema de Análises Estatísticas e Genética, versão 5.0, da Universidade Federal de Viçosa ${ }^{20}$.

\section{RESULTADOSE DISCUSSÃO}

Após os exames hematológicos, as crianças que apresentavam anemia foram submetidas a tratamento medicamentoso com sulfato ferroso, prescrito por um médico pediatra da Prefeitura Municipal de Viçosa, MG. Na Tabela 1, encontra-se a prevalência de anemia observada nas três creches estudadas.

As crianças anêmicas, sob medicação, foram excluídas das análises seguintes, portanto não constaram na amostragem do período final, novembro de 2001. Foram, ainda, excluídas do grupo de avaliação as crianças que saíram da creche ou não consumiram toda a porção oferecida dos produtos testados. Das 53 crianças selecionadas no início do estudo, em 36 crianças remanescentes, procedeu-se aos mesmos exames bioquímicos, dietéticos e antropométricos feitos antes da intervenção.

A distribuição, realizada de segunda a sexta-feira, foi feita durante 70 dias, com mediana de 41. As médias de freqüência das crianças nas creches foram de 35,55, com desvio-padrão (dp) de 5,64 dias na creche $A$, na qual foi fornecida refeição sem acréscimo de ferro; 38,83 dp=5,17

Tabela 1. Prevalência de anemia em três creches municipais de Viçosa, MG, segundo a faixa etária, no período inicial do estudo. Agosto de 2001.

\begin{tabular}{lccccc}
\hline \multirow{2}{*}{$\begin{array}{l}\text { Faixa etária } \\
\text { (meses) }\end{array}$} & $\begin{array}{c}\text { Creches } \\
(n=13)\end{array}$ & \begin{tabular}{c} 
B \\
\cline { 2 - 4 }
\end{tabular} & $\begin{array}{c}\text { C } \\
(n=17)\end{array}$ & $\begin{array}{c}\text { Total de } \\
\text { anêmicos }\end{array}$ & $\begin{array}{c}\text { Anêmicos } \\
(\%)\end{array}$ \\
\hline $24-35(n=4)$ & 0 & 1 & 0 & 1 & 25,0 \\
$36-47(n=12)$ & 2 & 0 & 0 & 2 & 16,67 \\
$48-59(n=25)$ & 2 & 1 & 2 & 5 & 20,0 \\
\hline Total & 4 & 2 & 2 & 8 & 15,09 \\
\hline
\end{tabular}


dias na creche $B$, em que se ofereceu alimentação com adição de sulfato ferroso; e 42,20 dp=4,95 dias na creche $C$, na qual foi fornecida multimistura. As médias de consumo de ferro, proveniente dos produtos distribuídos, foram de 0,230 mg/dia, $0,595 \mathrm{mg} / \mathrm{dia}$ e $0,629 \mathrm{mg} / \mathrm{dia}$, nas creches sem adição de ferro, com sulfato ferroso e multimistura, respectivamente. As crianças da creche $\mathrm{A}$ (sem adição de ferro) apresentaram menor consumo de ferro proveniente dos produtos, porque os mesmos não foram fortificados com ferro; ofereciam apenas o ferro proveniente dos ingredientes que os compunham. As crianças das creches B (sulfato ferroso) e C (multimistura) tiveram semelhante ingestão diária de ferro proveniente dos produtos fornecidos, indicando que esses produtos divergiam apenas quanto à fonte de ferro administrada.

Os índices de peso/idade, peso/estatura e estatura/idade foram analisados neste período; $e$ foi observado que as crianças da creche A (sem adição de ferro) não apresentaram alterações nesses índices (Tabela 2, p>0,05). Nas crianças das creches $B$ (sulfato ferroso) e $C$ (multimistura) foram observadas alterações desses índices. $\mathrm{Na}$ creche do sulfato ferroso (creche B) houve redução significativa no índice estatura/idade, indicando que as crianças não tiveram incremento estatural adequado para a idade no período estudado $(p<0,05)$, podendo ser reflexo da deficiente ingestão dietética observada. Nas crianças da creche em que se forneceu multimistura, foi observada melhoria no índice peso/estatura, piorando, entretanto, o índice estatura/idade. Sabe-se que o incremento da estatura é proporcionalmente mais lento que o de peso. Assim, um déficit estatural tende a se desenvolver quando o agravo nutricional ocorre por tempo prolongado, podendo, ou não, ser recuperado por meio de alimentação adequada ${ }^{21}$.

Para os dados dos inquéritos dietéticos, foi avaliado se a média de ingestão, por nutriente, manteve-se constante durante o período de estudo. De acordo com os dados apresentados na Tabela 3 , pode-se observar que na creche $A$ (sem adição de ferro, $n=9$ ) apenas a média de ingestão de
Tabela 2. Diferença média entre os índices antropométricos peso/estatura, peso/idade e estatura/idade, em escore-Z, avaliados no início e final do estudo, nas crianças das creches municipais de Viçosa. Agosto a novembro de 2001.

\begin{tabular}{lccc}
\hline Creches & Peso/Estatura & Peso/Idade & Estatura/ldade \\
\hline A (sem adição de & 0,07 & $-0,06$ & $-0,13$ \\
ferro) & & & \\
B (sulfato ferroso) & 0,17 & 0,02 & $-0,35^{*}$ \\
C (multimistura) & $0,25^{*}$ & 0,12 & $-0,16^{*}$ \\
\hline
\end{tabular}

* Significante a $5 \%$ de probabilidade, pelo teste " $t$ " pareado.

vitamina $C$ foi superior $(p<0,05)$ no final do estudo. As médias de ingestão dos demais nutrientes não sofreram alterações no período estudado $(p>0,05)$. $\mathrm{Na}$ creche $B$ ( sulfato ferroso, $n=12$ ), observou-se que as ingestões de vitaminas $A$ e $C$ sofreram alteração no período estudado, sendo maior no início da pesquisa $(p<0,05)$. Nos demais nutrientes foi mantida a média de ingestão durante o experimento, sendo semelhante às médias iniciais e finais observadas $(p>0,05)$. Na creche multimistura ( $C, n=15)$, observaram-se aumento no consumo de cálcio e redução na ingestão de fibras ao final do estudo $(p<0,05)$. Nos demais nutrientes, as médias de ingestão mantiveram-se semeIhantes $(p>0,05)$ durante o período experimental.

Esses dados de ingestão são de grande importância na análise dos resultados hematológicos, uma vez que a dieta fornece tanto inibidores quanto promotores da absorção de ferro, além de ser determinante do estado nutricional do indivíduo. Assim, a ingestão de nutrientes reguladores da biodisponibilidade de ferro esteve comprometida nas três creches estudadas. $\mathrm{O}$ teor médio de vitamina $C$ ingerido, um importante promotor da absorção de ferro ${ }^{22}$, ficou muito aquém das necessidades nos dois períodos estudados. O ferro também estabelece interações negativas com constituintes dietéticos, como cobre, zinco e cálcio, com os quais compete pelo sítio de ligação para absorçãa ${ }^{23}$. Neste estudo, esses quatro minerais apresentaram ingestão insuficiente, em comparação com a recomendada, indicando que, fisiologicamente, poderia estar 
ocorrendo um estado carencial mais abrangente que uma simples deficiência de ferro.

Com relação ao consumo diário de ferro, todas as crianças, nas três creches, apresentaram um baixo consumo, relativo à faixa etária em estudo ${ }^{16}$. Ainda foi possível observar um menor consumo de ferro, em todas as creches ao final do estudo, entretanto, sem diferença estatística significativa (Tabela 3, p>0,05). A creche A (sem adição de ferro) mostrou uma média de consumo de ferro de 6,17 dp=1,9mg/dia no início do estudo; e, de 5,03 dp=1,6mg/dia ao final. A creche B (sulfato ferroso) mostrou uma média de 7,55 $\mathrm{dp}=2,15 \mathrm{mg} /$ dia no início; e, de 6,51 dp=2,34mg/ dia ao final do estudo. $E$, na creche $C$ (multimistura), média de 7,06 dp=1,66mg/dia no início, e $6,59 \mathrm{dp}=1,77 \mathrm{mg} / \mathrm{dia}$ no final. Assim, observa-se que as médias de ingestão de ferro estavam muito aquém das necessidades fisiológicas das crianças $^{16}$. Considerando, ainda, que os produtos fornecidos para as creches B (sulfato ferroso) e C (multimistura) cobriam cerca de $10 \%$ das recomendações de ferro para essa faixa etária, essa quantidade não seria, portanto, capaz de suprir as necessidades desse nutriente, ficando a dieta habitual da criança com maior contribuição em seu estado de ferro orgânico.

Tabela 3. Diferença média de ingestão, por nutriente, em cada creche estudada do município de Viçosa, 2001

\begin{tabular}{lccc}
\hline & \multicolumn{3}{c}{ Creches } \\
\cline { 2 - 4 } Nutrientes & $\begin{array}{c}\text { A } \\
\text { (sem adição de } \\
\text { ferro) }\end{array}$ & $\begin{array}{c}\text { B } \\
\text { (sulfato } \\
\text { ferroso) }\end{array}$ & $\begin{array}{c}\text { C } \\
\text { (multimistura) }\end{array}$ \\
\hline Energia (Cal) & $-69,16$ & $-99,86$ & 98,86 \\
Carboidratos (g) & $-7,55$ & $-22,21$ & 15,27 \\
Lipídios (g) & $-2,94$ & 0,85 & 1,02 \\
Proteínas (g) & $-3,18$ & $-4,46$ & 1,21 \\
Ferro (mg) & $-1,14$ & $-1,07$ & $-0,46$ \\
Cálcio (mg) & 16,35 & $-96,32$ & 114,81 * \\
Zinco (mg) & 0,71 & $-0,21$ & 0,55 \\
Cobre (mg) & 0,05 & 0 & $-0,05$ \\
Vitamina C (mg) & 12,36 * & $-32,56 *$ & $-4,01$ \\
Vitamina A (mg) & 48,65 & $-129,42 *$ & 52,95 \\
Fibra (g) & 0,83 & 0,54 & $-1,88 *$ \\
\hline
\end{tabular}

*Significante a $5 \%$ de probabilidade, pelo teste " $t$ " pareado.
Além de avaliar a adequação dietética, principalmente da ingestão de ferro, como um fator de risco para a instalação de um quadro de anemia, foram também analisados os parâmetros hematológicos e bioquímicos. Foram utilizados parâmetros laboratoriais variados para avaliar o ferro em estoque e circulante. No período inicial, foi realizada uma avaliação hematológica, a partir da qual foram selecionadas as crianças, não-anêmicas $(\mathrm{Hb}>11,0 \mathrm{~g} / \mathrm{dl})$, que seriam avaliadas no período final.

Na Tabela 4 verifica-se que as crianças da creche $\mathrm{A}$ (sem adição de ferro) mantiveram seus índices bioquímicos inalterados durante o período avaliado, uma vez que não foi detectada diferença $(p>0,05)$ entre os valores iniciais e finais de hemoglobina, hematócrito, hemácias e ferritina. Entretanto, a média de concentração de ferro sérico foi superior ao final do estudo $(p<0,05)$. Os índices nas crianças da creche que receberam sulfato ferroso (creche B) e multimistura (creche C) como fonte de ferro sofreram alterações no período estudado $(p<0,05)$, exceto a ferritina, que manteve seus índices inalterados $(p>0,05)$. Foi observada redução nos níveis de hemoglobina, hematócrito e hemácias; entretanto, o nível de ferro sérico sofreu aumento $(p<0,05)$. Esses resultados não refletiram a expectativa, uma vez que as creches que receberam ferro apresentaram redução dos índices analisados. Tal fato pode estar ligado a diversos fatores, principalmente dietéticos. Na creche A (sem adição de ferro) foi observado aumento na ingestão de vitamina $\mathrm{C} e$ na creche $B$ (sulfato ferroso), redução da ingestão dessa vitamina, promotora da absorção de ferro. Já na creche que recebeu multimistura foi observado maior consumo de cálcio, um competidor de absorção com o ferro. Esses fatores não estavam sendo controlados e seguiram o padrão dietético próprio da amostra estudada, entretanto deveriam ter sido homogêneos para que as variações entre os grupos pudessem ter sido menores, diminuindo a interferência no aproveitamento orgânico de ferro.

Com relação à ferritina e ao ferro sérico, as médias apresentadas na Tabela 4 retratam um 
estado de normalidade, próximo à deficiência, para tais parâmetros. No período estudado, os níveis de ferritina permaneceram inalterados, evidenciando-se que os produtos fornecidos não influenciaram, efetivamente, no fornecimento de ferro ao organismo, uma vez que não houve recuperação de suas reservas. Associado à pequena quantidade de ferro fornecida pelos produtos de panificação, a ingestão de ferro permaneceu deficiente (Tabela 3), não sendo capaz de recuperar o estado de ferro, já inicialmente deficiente, o que impossibilitou uma melhor avaliação das crianças em estudo. Entretanto, foi observado aumento no ferro sérico, o que indicaria a existência de ferro disponível para a eritropoiese normal. Uma vez observado que os níveis de hemoglobina sofreram redução significativa nas crianças das creches do sulfato ferroso e multimistura $(p<0,05)$, os resultados de ferro sérico são incompatíveis com o estado de ferro circulante.

Vários estudos têm reportado a incontestável inibição exercida por algumas substâncias químicas sobre a absorção de ferro não-heme, proveniente de alimentos de origem vegetal. Entre essas substâncias, citam-se fitatos, oxalatos, taninos e polifenóis, que formam complexos insolúveis com o ferro na luz intestinal ${ }^{24,25}$. A multimistura em questão é fonte de quantidades apreciáveis de fitato e oxalato, estabelecendo-se razões molares, com cátions bivalentes, como o ferro, o cálcio e o zinco, problemáticos do ponto de vista nutricional ${ }^{8}$. Preocupa sua utilização, uma vez que, neste caso, tem-se a associação de um reduzido consumo de ferro e a presença desses fatores antinutricionais. Conrad ${ }^{3} \mathrm{fez}$, ainda, referência ao efeito inibitório que o cálcio exerce sobre a absorção de ferro, tanto heme quanto não-heme, sendo mais um fator preocupante na faixa etária em questão, considerando o padrão dietético próprio da idade, que emprega alimentos lácteos em grandes quantidades.

Vítolo et al. ${ }^{21}$ reportaram que a carência nutricional de ferro está associada às condições de desenvolvimento e alimentação do país, uma vez que a prevalência de anemia em crianças norte-americanas, menores de 5 anos, é de $3 \%$. Oyarzún et al. ${ }^{26}$, em estudo teórico para melhorar a adequação nutricional de vitaminas e minerais, afirmaram que, dificilmente, ocorrerá adequação de ferro e zinco sem a adição de alimentos de origem animal. Entretanto, esse dado não é real para muitos países e nações do mundo, como o Brasil. O fato de incorporar uma fonte de ferro não-hemínico na dieta, como os provenientes de fontes de origem vegetal, também não quer dizer que irá ocorrer adequação nutricional desse mineral. Além da quantidade, deve-se avaliar a biodisponibilidade do ferro procedente de alimentos vegetais. Os alimentos alternativos aqui estudados, apesar de serem fontes de ferro, fornecem os inibidores de sua absorção. Os resultados levam a crer que nas crianças, já com níveis depletados de ferro, a ausência de um fornecimento dietético eficiente acelerou a implantação da deficiência orgânica desse mineral.

As crianças da creche A (sem adição de ferro) tinham o desenvolvimento mais comprometido, o que pode ter implicado menores necessidades fisiológicas de ferro (Tabela 4 , $p>0,05)$, em que, apesar de estarem próximos à deficiência, não houve piora do quadro geral. Já as crianças das creches B (sulfato ferroso) e C (multimistura) tiveram comportamento diferente, mesmo recebendo os produtos contendo ferro. Foi observado, no período estudado, que o índice estatura/idade nas creches B e C sofreu redução, podendo indicar que a deficiência dietética influenciou, de forma mais acentuada, essas crianças.

Este estudo, então, enfatiza a pluralidade de causas que cercam o estado nutricional de um indivíduo, mas não comprovou efeito das diferentes fontes de ferro, dado que as creches que receberam suplementação apresentaram redução dos níveis hematológicos (Tabela 4, p<0,05), o que não ocorreu na creche que não recebeu adição de ferro (creche A), que não tinha fortificação de 
Tabela 4. Parâmetros bioquímicos analisados nas crianças das creches do município de Viçosa, no início e final do estudo, 2001 ** .

\begin{tabular}{|c|c|c|c|c|c|c|}
\hline Creches & & $\mathrm{Hb}^{\mathrm{a}}(\mathrm{g} / \mathrm{dl})$ & $\mathrm{Ht}^{\mathrm{b}} \quad(\%)$ & $\mathrm{Hm}^{\mathrm{a}}$ (milhões $/ \mathrm{mm}^{3}$ ) & Ferritina $^{a}(\eta g / m l)$ & Fe Sérico $(\mu \mathrm{g} / \mathrm{dl})$ \\
\hline A & Inicial & $11,64 \pm 0,38$ & 35,200 & $4,35 \pm 0,34$ & $29,41 \pm 15,51$ & $59,45 \pm 22,48$ \\
\hline \multirow[t]{3}{*}{ (sem adição de ferro) } & Final & $11,50 \pm 0,74$ & 35,000 & $4,47 \pm 0,27$ & $33,69 \pm 18,26$ & $82,59 \pm 28,33$ \\
\hline & Diferença & $-0,14$ & $-0,020$ & 0,12 & 4,28 & 23,14 \\
\hline & $\mathrm{P}$ & 0,2133 & 0,383 & 0,1990 & 0,1389 & 0,0107 * \\
\hline B & Inicial & $11,88 \pm 0,52$ & 36,100 & $4,49 \pm 0,25$ & $23,08 \pm 11,63$ & $62,98 \pm 15,34$ \\
\hline \multirow[t]{3}{*}{ (sulfato ferroso) } & Final & $11,58 \pm 0,70$ & 34,150 & $4,39 \pm 0,33$ & $20,67 \pm 5,80$ & $84,49 \pm 23,08$ \\
\hline & Diferença & $-0,30$ & $-1,950$ & $-0,09$ & $-2,41$ & 21,51 \\
\hline & P & $0,0295^{*}$ & $0,020 *$ & $0,0452 *$ & 0,1525 & $0,0029 *$ \\
\hline C & Inicial & $12,05 \pm 0,90$ & 36,500 & $4,61 \pm 0,37$ & $24,32 \pm 21,51$ & $61,09 \pm 24,79$ \\
\hline \multirow[t]{3}{*}{ (MM) } & Final & $11,58 \pm 0,88$ & 35,200 & $4,45 \pm 0,27$ & $21,03 \pm 18,87$ & $79,06 \pm 15,93$ \\
\hline & Diferença & $-0,47$ & $-1,300$ & $-0,15$ & $-3,29$ & 17,97 \\
\hline & $\mathrm{P}$ & $0,0003^{*}$ & $0,002 * *$ & $0,0077^{*}$ & 0,2374 & $0,0076^{*}$ \\
\hline
\end{tabular}

$\mathrm{Hb}=$ hemoglobina; $\mathrm{Ht}=$ hematócrito; $\mathrm{Hm}=$ hemácias; $\mathrm{MM}=$ multimistura; ${ }^{a}$ Média \pm desvio-padrão= teste " $t$ " pareado; ${ }^{\mathrm{b}} \mathrm{Mediana}=$ teste de Wilcoxon; *Significante; **agosto a novembro.

ferro nos produtos testados. As crianças, de maneira geral, apresentaram ingestão deficiente de ferro ao longo do período estudado, o que foi refletido nos índices bioquímicos avaliados, que tinham seus valores muito próximos aos limites inferiores para a faixa etária.

Em ambas as creches suplementadas as crianças tiveram os índices de hemoglobina, hematócrito e hemácias reduzidos. Pôde-se observar que as crianças dessas creches tiveram um reduzido consumo de ferro durante todo o estudo (Tabela 3); e que, as crianças da creche que ingeriram multimistura podem ter sofrido uma depleção mais acentuada, em função de terem recebido, também, os fatores antinutricionais presentes na multimistura utilizada; ou devido ao aumento do consumo de cálcio. Assim, pode ser retomada a questão da quantidade de ferro fornecida como suplementação, cerca de $1 \mathrm{mg} /$ dia, proveniente dos produtos fornecidos. Essa quantidade não alterou o nível de ingestão dietética de ferro pelas crianças e foi insuficiente para melhorar os índices hematológicos avaliados.

\section{CONCLUS Ã O}

Este estudo não comprovou efeito das diferentes fontes de ferro (sulfato ferroso ou multimistura), dado que as creches que receberam suplementação apresentaram redução dos níveis hematológicos avaliados $(p<0,05)$, o que não ocorreu com a creche que não recebeu adição de ferro. Alguns fatores contribuíram para esse resultado, como o crescimento das crianças e a ingestão de alimentos durante o período estudado. Uma alimentação deficiente em ferro, em um período biológico de elevada demanda orgânica desse mineral, pode ter favorecido o estado de depleção de ferro observado nas crianças em estudo.

A quantidade de ferro fornecida como suplementação (cerca de $1 \mathrm{mg} / \mathrm{dia}$ ), proveniente dos produtos, não alterou o nível de ingestão dietética de ferro pelas crianças, e foi insuficiente para melhorar os índices hematológicos avaliados. Em se tratando de crianças que já tinham nível expressivo de comprometimento no estado nutricional relativo ao ferro, a avaliação da quantidade ingerida de ferro por dia torna-se, talvez, mais importante que a avaliação da fonte de ferro utilizada, sulfato ferroso ou multimistura, limitando-se as conclusões quanto à utilização da multimistura em estudo.

\section{A GRADECIMENTO}

À concessão da bolsa de estudo pelo Conselho Nacional de Desenvolvimento Científico e Tecnológico (CNPq). 


\section{REFERÊ NCIAS}

1. Paiva AA, Rondó PHC, Guerra-Shinohara EM. Parâmetros para avaliação do estado nutricional de ferro. Rev Saúde Pública. 2000; 34(4):421-26.

2. Davidsson L, Ziegler EE, Kastenmayer P, Hurrell RF. Erythrocyte incorporation of iron by infants: iron bioavalability from a low-iron infant formula and an evaluation of the usefulness of correcting erythrocyte incorporation values, using a reference dose or plasma ferritin concentrations. $\mathrm{Br} J$ Nutr. 2000; 84(5):847-53.

3. Conrad ME. Iron deficiency anemia. eMedical J [Internet]. 2002. [cited 2002 Feb 19]; 3(2). Available from: http://www.emedecine.com

4. Del Ciampo LA, Almeida CAN. Avaliação do estado nutricional de ferro na criança. Rev Paul Pediatr. 2002; 20(1):37-42.

5. Conrad ME. Anemia. eMedical J [Internet]. 2002. [cited 2002 Feb 15]; 3(2). Available from: http:// www.emedicine.com

6. Simões MCC, Moura EC, Sgarbieri VC, Figueiredo DB. Avaliação do impacto de um suplemento nutricional rico em ferro hematínico. Cad Saúde Pública. 1999; 15(4):871-81.

7. Brandão $C T$, Brandão RF. Alimentação alternativa. Brasília: Centro de Pastoral Popular; 1996.

8. Sant'Ana LFR, Costa NMB, Oliveira MGA, Gomes MRA. Valor nutritivo e fatores antinutricionais de multimisturas utilizadas como alternativa alimentar. Br J Food Technol. 2000; 3:129-35.

9. World Health Organization. Nutritional anaemias. Geneva; 1968. Technical Report Series, 405.

10. Pao EM, Cypel YS, Cálculo da ingesta alimentaria. In: Ziegler EE, Filer Júnior LJ. Conocimientos actuales sobre nutrictión. 7.ed. Washington (DC): OPS; 1997

11. Pinheiro ABV, Lacerda EMA, Benzecry EH, Gomes MCS, Costa VM. Tabela para avaliação de consumo alimentar em medidas caseiras. 4.ed. São Paulo: Atheneu; 2001.

12. Monteiro JBR, Esteves E. Diet Pro: sistema de suporte à avaliação nutricional e prescrição de dietas [programa de computador]. Versão 2.0. Viçosa, MG: Universidade Federal de Viçosa; 1996.

13. World Health Organization Working Group. Use and interpretation of anthropometric indicators of nutritional status. Bull World Health Organ. 1986; 64(6):929-41.

14. Dean AG, Dean JA, Burton AH, Dicker RC. Epi Info: a word processing database, and statistics program for public health [computer program]. Version 6.01. Georgia: Center for Disease Control \& Prevention; 1990.

15. Jellife DB. The assessment of the nutritional status of the community. Geneva: WHO; 1966.

16. Food and Nutrition Board. Dietary reference intakes (DRIs): recommended intakes for individuals, elements. Washington (DC): Institute of Medicine; 2001.

17. Ferreira ACR, Sant'Ana LFR, Costa, NMB. Desenvolvimento e avaliação sensorial de produtos contendo multimistura para utilização em ensaio de biodisponibilidade de ferro em pré-escolares. In: Resumos do X Simpósio de Iniciação Científica; 2000; Viçosa. Viçosa: Universidade Federal de Viçosa; 2000.

18. Vieira S, Hoffmann R. Estatística experimental. São Paulo: Atlas; 1989

19. Siegel S. Estatística não-paramétrica (para as ciências do comportamento). São Paulo: McGraw-Hill do Brasil; 1975.

20. Sistema de Análises Estatísticas e Genéticas [programa de computador]. Desenvolvido pela equipe técnica da Fundação Arthur Bernardes. Versão 5.0. Viçosa, MG: Universidade Federal de Viçosa; 1993.

21. Vítolo MR, Aguirre ANC, Kondo MR, Giuliano Y, Ferreira N, Lopez FA. Impacto do uso de cereal adicionado de ferro sobre os níveis de hemoglobina e a antropometria de pré-escolares. Rev Nutr. 1998; 11(2):163-71.

22. Angelis RC, Ctenas MLB. Biodisponibilidade de ferro na alimentação infantil. São Paulo: Nestlé; 1993. Temas de Pediatria, n.52.

23. Dutra-de-Oliveira JE, Marchini JS. Ciências nutricionais. São Paulo: Savier; 1998.

24. Hurrell RF, Davidsson L, Reddy M, Kastenmayer P, Cook JD. A comparison of iron absorption in adults and infants consuming identical infant formulas. Br J Nutr. 1998; 79(1):31-6.

25.Zijp IM, Korver O, Tijburg LBM. Effect of tea and other dietary factors on iron absorption. Crit Rev Food Sci Nutr. 2000; 40(5):371-98.

26. Oyarzún MT, Uauy R, Olivares S. Enfoque alimentario para mejorar la adecuación nutricional de vitaminas y minerales. Arch Latinoam Nutr. 2001; 51(1):7-18.

Recebido em: 13/7/2004

Versão final reapresentada em: 25/2/2005 Aprovado em: 30/3/2005 